\title{
Digitization of Bézier Curves and Patches using Discrete Geometry
}

\author{
Oscar Figueiredo ${ }^{1}$, Jean-Pierre Reveillès ${ }^{2}$, Roger D. Hersch ${ }^{1}$ \\ ${ }^{1}$ Ecole Polytechnique Fédérale de Lausanne \\ EPFL/DI-LSP CH-1015 Lausanne (Switzerland) \\ \{Oscar.Figueiredo, RD.Hersch\}@epfl.ch \\ ${ }^{2}$ LLAIC1, Computer Science Institute of Technology \\ BP 86, Université d'Auvergne, Les Cézeaux 63720 Aubière (France) \\ reveil@llaic.u-clermont1.fr
}

\begin{abstract}
Existing algorithms for rendering Bézier curves and surfaces fall into two categories: iterative evaluation of the parametric equations (generally using forward differencing techniques) or recursive subdivision. In the latter case, all the algorithms rely on an arbitrary precision constant (tolerance) whose appropriate choice is not clear and not linked to the geometry of the image grid. In this paper we show that discrete geometry can be used to improve the subdivision algorithm so as to avoid the need for any arbitrary value. The proposed approach extends well and we present its application in the case of 2D and 3D Bézier curves as well as Bézier triangle patches and tensor-product surface patches.
\end{abstract}

Keywords. Bézier curves, Bézier surfaces, De Casteljau subdivision, polygonalization

\section{Introduction}

Bézier curves and patches are among the most fundamental primitives in computer graphics and computer aided modeling. However, as they are defined by means of mathematical equations, they are continuous objects which are not ideally suited for a computer representation. Discrete geometry aims at providing an equivalent of these mathematical objects in the same way as it has established formal definitions of digital lines and planes [9]. A first step towards this goal consists in developing a discretization algorithm of continuous Bézier curves and patches which would be consistent with existing results in discrete geometry. In this paper, we propose such an algorithm. Our approach is based on a classical De Casteljau recursive subdivision algorithm but with a new flatness criterion based on the digital geometry of lines and planes which guarantees a recursion depth close to optimal and an appropriate connectivity of the obtained discrete curve or surface with no need for arbitrary constants. Moreover, though we restrict our presentation here to the case of cubic Bézier curves and patches for the sake of clarity, it is a remarkable fact that the approach is general enough to be easily extended to higher degrees and dimensions. 


\section{Discrete lines and planes}

It has been shown that beyond an algorithmic description, digital lines and planes can be given rigorous mathematical formulations. Thus a planar digital line is a subset of - 2 , denoted $D(\mathbf{n}, \gamma, \rho)$ described by a diophantine equation of the following form :

$$
D(\mathbf{n}, \gamma, \rho): \quad \gamma \leq n_{x} x+n_{y} y<\gamma+\rho
$$

where $\left(n_{x}, n_{y}\right) \in \mathcal{C}^{2}$ defines the direction of the line, $\gamma \in \mathcal{}$ defines its affine offset while $\rho \in$ ' is called arithmetical thickness. A particularly interesting subset of digital lines consists of those verifying $\rho=\max \left(\left|n_{x}\right|,\left|n_{y}\right|\right)$, which are simply denoted $D(\mathbf{n}, \gamma)$ :

$$
D(\mathbf{n}, \gamma): \quad \gamma \leq n_{x} x+n_{y} y<\gamma+\max \left(\left|n_{x}\right|,\left|n_{y}\right|\right)
$$

which are called naive digital lines and have exactly the same structure as the sets drawn by the classical Bresenham algorithm for lines [1]. The most important property of naive lines is strict 8-connectivity.

$D(\mathbf{n}, \gamma)$ is the digitization by truncation of the ordinary euclidean line of equation $n_{x} x+n_{y} y=\gamma$ while $D\left(\mathbf{n}, \gamma-\left[\frac{\max \left(\left|n_{x}\right|,\left|n_{y}\right|\right)}{2}\right]\right)$ is its digitization by the closest integer point.

When considered in ? ${ }^{2}$ instead of - 2 , Equation 2 defines a continuous strip of width (measured orthogonally to the direction of the strip) $w=\frac{\max \left(\left|n_{x}\right|,\left|n_{y}\right|\right)}{\sqrt{n_{x}^{2}+n_{y}^{2}}}$ which can be thought of as the continuous counterpart of the naive digital line. Such a width $w$ corresponds to a thickness of 1 in the direction perpendicular to the main line direction.

Digital planes are defined by a similar diophantine equation and thus share similar properties. We call digital plane the subset of ${ }^{-3}$ denoted $P(\mathbf{n}, \gamma, \rho)$ that verifies the following equation:

$$
P(\mathbf{n}, \gamma, \rho): \quad \gamma \leq n_{x} x+n_{y} y+n_{z} z<\gamma+\rho .
$$

In the same way as naive digital lines, digital planes verifying $\rho=\max \left(\left|n_{x}\right|,\left|n_{y}\right|,\left|n_{z}\right|\right)$ are called naive digital planes and are simply denoted $P(\mathbf{n}, \gamma)$. Naive digital planes are 18 -connected sets with no holes for 6-connectivity. One of the most fundamental properties of digital planes is their being functional along the main direction of their normal, i.e., if $n_{z}=\max \left(\left|n_{x}\right|,\left|n_{y}\right|,\left|n_{z}\right|\right)$ then for each $(x, y)$ there exists a single $z$ such that $(x, y, z)$ belongs to the digital plane. 
In addition, $P(\mathbf{n}, \gamma)$ is the digitization by truncation of the ordinary euclidean plane of equation $n_{x} x+n_{y} y+n_{z} z=\gamma$.

Similar to euclidean lines in ? ${ }_{n}^{3}$ which are commonly defined as the intersection of two euclidean planes, three-dimensional digital lines can be conveniently represented by the intersection of two particular digital planes though such a definition is not ideal from a theoretical point of view [5]. Thus a digital line of direction $\mathbf{n} \in{ }^{-3}$ with $n_{z}=\max \left(\left|n_{x}\right|,\left|n_{y}\right|,\left|n_{z}\right|\right)$, denoted $D(\mathbf{n}, \gamma, v)$ is the set of points $(x, y, z)$ of ${ }^{3}$ verifying:

$$
D(\mathbf{n}, \gamma, v): \quad\left\{\begin{array}{l}
\gamma \leq-n_{z} x+n_{x} z<\gamma+n_{z} \\
v \leq-n_{z} y+n_{y} z<v+n_{z}
\end{array} .\right.
$$

When considered in ? ${ }_{1}^{3}$ instead of -3 , Equation 4 defines a generalized continuous cylinder having for intersection with the plane $x O y$ (main plane orthogonal to the axis $O z$ along which the direction of the line has its biggest coordinate) a unit square. Thus a digital 3D line can be seen as the set of integer points contained in such a cylinder.

\section{Digitization of cubic Bézier curves}

There exists essentially two different approaches to the scan-conversion of Bézier curves and surface patches [6]. The first one uses the parametric representation of the curve and evaluates repetitively the equations using forward differencing. Forward differencing is a fast and efficient technique which can be hardware accelerated. However it suffers from two major drawbacks: first, it is a floating point algorithm subject to numerical drifts due to error accumulation [2] and whose implementation requires great care and a register width depending on the number of pixels to draw, furthermore there is naturally no linear relation between the parameter and the coordinates of the drawn points. Hence a regular subdivision of the parameter interval, though simple, is particularly inefficient since it can lead to many unneeded evaluations, drawing the same discrete point (if the parameter interval is too small) or holes in the curve, respectively patch, (if the parameter interval is too big). Therefore refinements such as dynamic step size adjustment are preferred $[2,4,10]$. But even in that case there is still a need for choosing a parameter increment and criterions for deciding when to scale that increment [4]. Unfortunately all those criterions are based on some geometrical value (surface of a triangle, distance between a point and a line, angle) being "small" and thus require determining a tolerance constant which, in practice is often chosen arbitrarily and whose relation to the sampling grid is not clear.

The second approach uses the De Casteljau algorithm [3], a stable and efficient method with intrinsic adaptiveness to the curve. This method consists in recursively subdividing the control polygon $\left(P_{0}^{0}, P_{1}^{0}, P_{2}^{0}, P_{3}^{0}\right)$ into two sub-polygons 
$\left(P_{0}^{0}, P_{0}^{1}, P_{0}^{2}, P_{0}^{3}\right)$ and $\left(P_{0}^{3}, P_{1}^{2}, P_{2}^{1}, P_{3}^{0}\right)$ (Figure 1) where the $P_{j}^{i}$ are defined as weighted sums of polygon vertices:

$$
P_{i}^{n+1}=\alpha P_{i}^{n}+(1-\alpha) P_{i+1}^{n} \quad \alpha \in[0,1] .
$$

At each step the area of a new control polygon is smaller and hence is closer to the Bézier arc which remains invariant along the process. The recursion can be stopped when the control polygon is close enough to the curve.

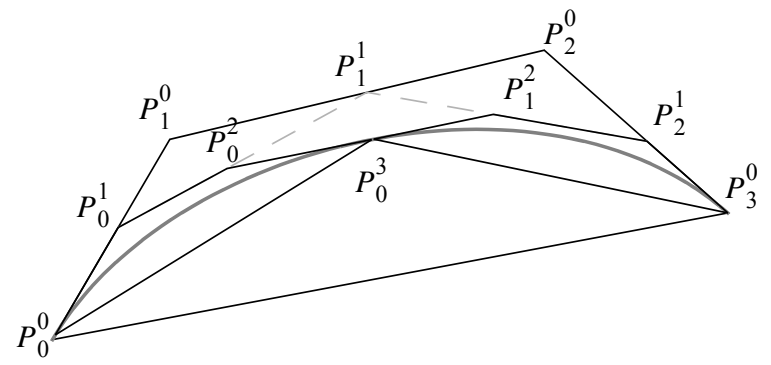

Figure 1: De Casteljau Subdivision

Theorems exist that indicate when the maximum distance between the arc and the control polygon is smaller than $\varepsilon$, based on the geometry of the sub-polygons $[8,11]$ or directly based on the initial control polygon and the recursion depth [7]. All of these results rely on the choice of an ad hoc constant $\varepsilon$ which makes them quite unsatisfying from a theoretical point of view. In what follows we show that we can eliminate the need for such a constant.

We denote with $[r]$ the integer part of $r \in$ ? ", i.e., the greatest integer smaller than $r$. Similarly we denote with $[R]$ the integer point of -2 (resp. ${ }^{-3}$ ) whose coordinates are the respective integer parts of the coordinates of $R \in$ ? ${ }^{2}$ (resp. ? " ${ }^{3}$ ). Let us consider an arc of integer endpoints $(P, Q)$. We call axis of the arc, the line defined by its two endpoints. The vector PQ is the direction of the arc. We also call width of the arc, the diameter of the smallest cylinder of axis $(P, Q)$ that encompasses the whole arc. And similarly we call width of a set of points of ? " ${ }^{2}, E=\left\{P_{i}\right\}_{0 \leq i<n}$ with respect to the direction $\left(n_{x}, n_{y}\right) \in-2$, the diameter of the narrowest cylinder of axis $\left(n_{x}, n_{y}\right)$ that encompasses $E$. This width $w_{\left(n_{x}, n_{y}\right)}(E)$ is given by:

$$
w_{\left(n_{x}, n_{y}\right)}(E)=\frac{\operatorname{dia}\left(\left\{n_{x} P_{i x}+n_{y} P_{i y}\right\}_{0 \leq i<n}\right)}{\sqrt{n_{x}^{2}+n_{y}^{2}}}
$$

where $\operatorname{dia}(A)$ for a subset $A$ of ? „is defined as:

$$
\operatorname{dia}(A)=\max _{a \in A}(a)-\min _{a \in A}(a) .
$$


Theorem 1. Let $C$ be a planar arc of integer endpoints $P, Q$ and of width $w$. If $w \leq \frac{\max \left(\left|\mathbf{P} \mathbf{Q}_{x}\right|,\left|\mathbf{P Q}_{y}\right|\right)}{\|\mathbf{P Q}\|}$ then the best 8-connected integer approximation of $C$ is a naive digital straight line segment of direction $\mathbf{P Q}$.

Proof. Equation 2 defines a naive digital line as the set of integer points contained in a continuous strip of the euclidean plane. The width $w$ of this euclidean strip relates to the arithmetic thickness of the digital line $\rho$ through the relation $w=\frac{\rho}{\sqrt{n_{x}^{2}+n_{y}^{2}}}$. It becomes clear then, that if $C$ verifies $w \leq \frac{\max \left(\left|\mathbf{P} \mathbf{Q}_{x}\right|,\left|\mathbf{P Q}_{y}\right|\right)}{\|\mathbf{P Q}\|}$ then it fits within the real boundaries of a naive digital line and hence there cannot be a better integer approximation to $C$ than a naive digital straight line segment. $\mathrm{n}$

Theorem 2. Let $B$ be a planar cubic Bézier arc defined by its control polygon $\left(P_{0}, P_{1}, P_{2}, P_{3}\right), B$ can be optimally represented by a naive digital straight line segment of direction $\left[\mathrm{P}_{0} P_{3}\right]$ iff

$$
\operatorname{dia}\left(\left\{n_{x} P_{i x}+n_{y} P_{i y}\right\}_{0 \leq i \leq 3}\right)<\frac{4}{3} \max \left(\left|n_{x}\right|,\left|n_{y}\right|\right)
$$

where $n_{x}=\left[P_{3 y}\right]-\left[P_{0 y}\right]$ and $n_{y}=-\left[P_{3 x}\right]+\left[P_{0 x}\right]$

Proof. Wang's theorem states that $B$ lies at most $\frac{3}{4} \max \left(d\left(P_{1}\right) d\left(P_{2}\right)\right)$ away from its axis where $d\left(P_{i}\right)$ denotes the distance of point $P_{i}$ to the axis and that this bound is optimal [11]. Furthermore the convex hull containment property of Bézier curves states that $B$ lies entirely within its control polygon [3]. Therefore the width of $B$ is at most $3 / 4$ of the width of its control polygon with respect to the line $\left(\left[P_{0}\right]\left[P_{3}\right]\right)$, the width of the control polygon being given by $\frac{\operatorname{dia}\left(\left\{n_{x} P_{i x}+n_{y} P_{i y}\right\}_{0 \leq i \leq 3}\right)}{\sqrt{n_{x}^{2}+n_{y}^{2}}}$. The result then follows directly from Theorem 1. $\mathrm{n}$

A control polygon verifying Theorem 2 is said to be flat and the recursive subdivision can be stopped at that level. If the control polygon does not meet the criterion of Theorem 2 then it is subdivided into two sub-polygons according to (5). Equation 5 leaves a degree of liberty in the choice of $\alpha$. Optimizing this value at each recursion step yields a digitization with the minimum number of discrete segments. Such optimization however is beyond the scope of this paper and in practice $\alpha=\frac{1}{2}$ is the usual choice. 
Theorem 2 does not provide the complete equation of the digital line segment that is the best approximation of the spline arc but only its direction. The affine offset $\gamma$ must also be determined. The ideal value of $\gamma$ which yields a discretization by the closest integer corresponds to the midline (axis) of the narrowest cylinder of direction $\left(\left[P_{0}\right]\left[P_{3}\right]\right)$ enclosing the curve within its control polygon. In order to find an algebraic formulation of $\gamma$, we must distinguish two cases depending on whether the curve crosses the line $\left(\left[P_{0}\right]\left[P_{3}\right]\right)$ or not.

1. The curve does not cross $\left(\left[P_{0}\right]\left[P_{3}\right]\right)$. In this case the curve lies between the line $\left(\left[P_{0}\right]\left[P_{3}\right]\right)$ and a parallel at three fourths of the distance of the most distant point of the control polygon to that line (this may be $P_{1}$ or $P_{2}$ ). Assuming that point to be $P_{1}$, $\gamma$ becomes:

$$
\gamma=\left[\frac{3\left(n_{x} P_{1 x}+n_{y} P_{1 y}\right)+5\left(n_{x} P_{0 x}+n_{y} P_{0 y}\right)}{8}\right]-\left[\frac{\max \left(\left|n_{x}\right|,\left|n_{y}\right|\right)}{2}\right] .
$$

2. The curve crosses $\left(\left[P_{0}\right]\left[P_{3}\right]\right)$. In this case the curve is enclosed in a cylinder of direction $\left[\mathbf{P}_{\mathbf{0}} \mathbf{P}_{\mathbf{3}}\right]$ whose axis is midway between the points $P_{1}$ and $P_{2}$. Hence $\gamma$ becomes:

$$
\gamma=\left[\frac{\left(n_{x} P_{1 x}+n_{y} P_{1 y}\right)+\left(n_{x} P_{2 x}+n_{y} P_{2 y}\right)}{2}\right]-\left[\frac{\max \left(\left|n_{x}\right|,\left|n_{y}\right|\right)}{2}\right] .
$$

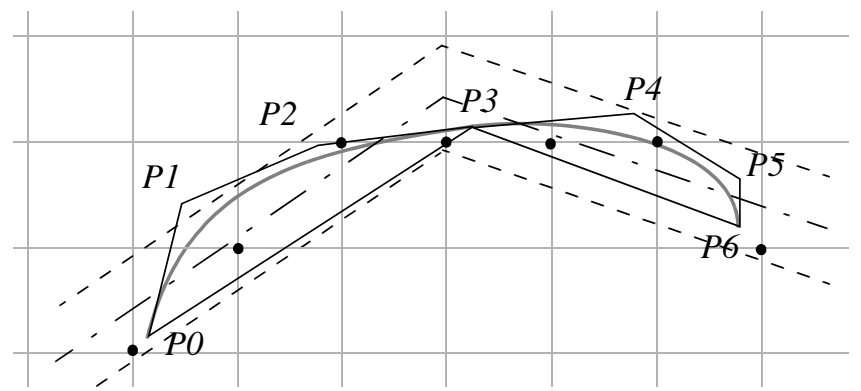

Figure 2: Discretization of a Bézier arc

A Bézier arc described by two flat control polygon $\left(P_{0}, P_{1}, P_{2}, P_{3}\right)$ and $\left(P_{3}, P_{4}, P_{5}, P_{6}\right)$. The round dots are the integer points making up the discretization of the Bézier arc. The dash-dotted lines represent the axes defined by Equation 9 while the simple dashed lines represent the real boundaries of the digital line segments as defined by Equation 2.

A criterion for digitizing 3D Bézier curves stems from the same principle as for 2D Bézier curves: the De Casteljau recursion stops and the curve segment can be rendered as a discrete line with no loss of precision when the convex hull of its control polygon is bounded by the limits of a 3D digital line as defined in (4). Denoting 
$n_{x}=\left[P_{3 x}\right]-\left[P_{0 x}\right], \quad n_{y}=\left[P_{3 y}\right]-\left[P_{0 y}\right], \quad n_{z}=\left[P_{3 z}\right]-\left[P_{0 z}\right] \quad$ and assuming $\left|n_{z}\right|=\max \left(\left|n_{x}\right|,\left|n_{y}\right|,\left|n_{z}\right|\right)$, the condition writes:

$$
\left\{\begin{array}{l}
\operatorname{dia}\left(\left\{-n_{z} P_{i x}+n_{x} P_{i z}\right\}_{0 \leq i \leq 3}\right) \leq \frac{4}{3}\left|n_{z}\right| \\
\operatorname{dia}\left(\left\{-n_{z} P_{i y}+n_{y} P_{i z}\right\}_{0 \leq i \leq 3}\right) \leq \frac{4}{3}\left|n_{z}\right|
\end{array} .\right.
$$

The affine offset proposed in Equations 9 and 10 still holds, with the same restrictions, in this case by considering independently the projections of the control polygon on the main planes $x O z$ and $y O z$.

\section{Digitization of 3D cubic Bézier surface patches}

We call naive digital plane patch a non-empty 26-connected subset of a naive digital plane $P(\mathbf{n}, \gamma)$ defined as a non-empty polygon on that plane by the following set of equations:

$$
Q\left(\mathbf{n}, \gamma,\left(\mathbf{q}_{k}, \lambda_{k}\right)_{0 \leq k<m}\right): \quad P(\mathbf{n}, \gamma) \cap\left\{\begin{array}{c}
q_{0 x} x+q_{0 y} y+q_{0 z} z<\lambda_{0} \\
q_{1 x} x+q_{1 y} y+q_{1 z} z<\lambda_{1} \\
\ldots \\
q_{(m-1) x} x+q_{(m-1) y} y+q_{(m-1) z} z<\lambda_{3}
\end{array}\right.
$$

where $\mathbf{q}_{k} \in{ }^{-3}$ and $\lambda_{k} \in$ - for $0 \leq k<m$.

Special interesting cases of naive digital plane patches include digital quadrilaterals $(m=4)$ and triangles $(m=3)$.

3D bicubic tensor-product Bézier patches as well as Bézier triangles can be approximated by naive digital plane patches as defined by Equation 12. Indeed the De Casteljau recursive construction is general and still applies in those cases.

Theorem 3. Let $B$ be a bicubic Bézier patch defined by its control net $\left(P_{i, j}\right)_{0 \leq j_{j} \leq 3}$. Let

$$
\begin{aligned}
\mathbf{u}= & {\left[\mathbf{P}_{\mathbf{0}, \mathbf{0}}\right]\left[\mathbf{P}_{\mathbf{3}, \mathbf{0}}\right], \mathbf{v}=\left[\mathbf{P}_{\mathbf{0 , 0}, \mathbf{0}}\right]\left[\mathbf{P}_{\mathbf{3}, \mathbf{3}}\right] \text { and } \mathbf{n}=\mathbf{u} \times \mathbf{v} \text { if } } \\
& \operatorname{dia}\left(\left\{n_{x}\left(P_{i, j}\right)_{x}+n_{y}\left(P_{i, j}\right)_{y}+n_{z}\left(P_{i, j}\right)_{z}\right\}_{0 \leq j_{j} \leq 3}\right) \leq \max \left(\left|n_{x}\right|,\left|n_{y}\right|,\left|n_{z}\right|\right)
\end{aligned}
$$

then the best 26-connected integer approximation of $B$ is a digital plane patch of normal direction $\mathbf{n}$. 
Theorem 4. Let $B$ be a Bézier triangular patch of degree d defined by its control net

$$
\begin{aligned}
& \left(P_{i j k}\right)_{\substack{0 \leq i, j, k \leq d \\
i+j+k=d}} \text { Let } \mathbf{u}=\left[\mathbf{P}_{\mathbf{0 0 3}}\right]\left[\mathbf{P}_{\mathbf{3 0 0}}\right], \mathbf{v}=\left[\mathbf{P}_{\mathbf{0 0 3}}\right]\left[\mathbf{P}_{\mathbf{0 3 0}}\right] \text { and } \mathbf{n}=\mathbf{u} \times \mathbf{v} \text { if } \\
& \quad \operatorname{dia}\left(\left\{n_{x}\left(P_{i j k}\right)_{x}+n_{y}\left(P_{i j k}\right)_{y}+n_{z}\left(P_{i j k}\right)_{z}\right\}_{\substack{0, j, k \leq d \\
i+j+k=d}}\right) \leq \max \left(\left|n_{x}\right|,\left|n_{y}\right|,\left|n_{z}\right|\right)
\end{aligned}
$$

then the best 26-connected integer approximation of $B$ is a digital plane triangle of normal direction $\mathbf{n}$.

Proof. Since the containment property of Bézier patches and triangles within their control net holds, Equations 13 and 14 being verified amounts to the whole surface $B$ lying between the limits defined by Equation 3 for a naive digital plane of direction $\mathbf{n}$. $\mathrm{n}$

The value of the affine offset $\gamma$ of the digital plane that represents the digitization of $B$ by the closest integer point is given by:

$$
\begin{aligned}
& \gamma=\left[\frac{\min \left(n_{x}\left(P_{i, j}\right)_{x}+n_{y}\left(P_{i, j}\right)_{y}+n_{z}\left(P_{i, j}\right)_{z}\right)_{0 \leq j}<3}{2}+\right. \\
&\left.\frac{\max \left(n_{x}\left(P_{i, j}\right)_{x}+n_{y}\left(P_{i, j}\right)_{y}+n_{z}\left(P_{i, j}\right)_{z}\right)_{0 \leq j_{j}^{i}<3}}{2}\right]-\left[\frac{\max \left(\left|n_{x}\right|,\left|n_{y}\right|,\left|n_{z}\right|\right.}{2}\right]
\end{aligned}
$$

\section{Connectivity issues}

\subsection{Connectivity of digitized Bézier curves}

The construction proposed previously for discretizing a 2D Bézier arc actually builds a continuous polygonal line (the dash-dotted line of Figure 2) whose discrete counterpart is the discretization of the Bézier curve. Thus the overall discretization forms an 8-connected path which is the most reasonable requirement for the discretization of a perfectly general Bézier arc. In simple cases, if the Bézier arc does not have too important changes in orientation, its overall discretization forms a 8-connected simple curve, i.e., every point of the discretization has exactly two 8-neighbors except for the endpoints (if the curve is not closed).

In the case of 3D Bézier curves the proposed construction does not create a continuous 3D polygonal 3D line since the midlines of consecutive segments may not be coplanar. However it can be shown that the overall discretization is still 26-connected.

Theorem 5. Let $D_{1}$ and $D_{2}$ be two digital $3 D$ lines as defined in Equation 4 and let $H_{1}$ and $H_{2}$ be their respective real square-based enclosing cylinders. If $H_{1} \cap H_{2} \neq \varnothing$ then $D_{1}$ and $D_{2}$ intersect or have two 26-adjacent points 
Proof. If $H_{1} \cap H_{2} \neq \varnothing$ then that intersection contains at least a real point $P_{0}\left(x_{0}, y_{0}, z_{0}\right)$. Since $P_{0}$ is contained in the enclosing cylinder $H_{1}$ of $D_{1}, D_{1}$ contains an integer point $I_{1}\left(X_{1}, Y_{1}, Z_{1}\right)$ such that

$$
\left\{\begin{array}{l}
\left|X_{1}-x_{0}\right|<1 \\
\left|Y_{1}-y_{0}\right|<1 \\
\left|Z_{1}-z_{0}\right|<1
\end{array}\right.
$$

A similar statement holds for $D_{2}$ which contains an integer point $I_{2}\left(X_{2}, Y_{2}, Z_{2}\right)$ such that

$$
\left\{\begin{array}{l}
\left|X_{2}-x_{0}\right|<1 \\
\left|Y_{2}-y_{0}\right|<1 \\
\left|Z_{2}-z_{0}\right|<1
\end{array}\right.
$$

Hence $I_{1}$ and $I_{2}$ are at least 26-adjacent if not equal which proves the theorem. $\mathrm{n}$

Each segment of 3D digital line making up the discretization of a 3D Bézier curve is the set of points contained in a truncated square-based cylinder (as mentioned in paragraph 2). Consecutive cylinders always have a non-void intersection since they contain at least one common real point (the point that is common to the two consecutive Bézier control polygons which is also part of the Bézier arc itself). Thus, thanks to Theorem 5, 26-adjacency between consecutive discrete straight line segments is guaranteed.

\subsection{Connectivity of digitized Bézier surface patches}

The connectivity problem becomes more intricate in the case of the digitization of Bézier surface patches where discrete connectivity has to be controlled along the whole length of the edges of patches. This involves determining the appropriate bounds of the equations defining the discrete plane patch in Equation 12. We examine the problem in the case of bicubic tensor product Bézier patches though the results still hold in the case of Bézier triangles. The problem is still mostly open in this case and we only provide directions towards formal proofs.

Let $B$ and $B^{\prime}$ be two adjacent Bézier bicubic tensor-product patches verifying Theorem 3 and having for respective control nets $\left(P_{i, j}\right)_{0 \leq j_{j}^{i} \leq 3}$ and $\left(P^{\prime}{ }_{i, j}\right)_{0 \leq{ }_{j}^{i} \leq 3}$

such that $P_{3, i}=P_{0, i}^{\prime}$ for $0 \leq i \leq 3$ (Figure 3).

Let $Q\left(\mathbf{n}, \gamma,\left(q_{k}, \lambda_{k}\right)_{0 \leq k \leq 3}\right)$ and $Q^{\prime}\left(\mathbf{n}^{\prime}, \gamma^{\prime},\left(q^{\prime}{ }_{k}, \lambda^{\prime}{ }_{k}\right)_{0 \leq k \leq 3}\right)$ be two naive digital plane patches and let us denote $H$ and $H^{\prime}$ their real enclosing parallelepipeds defined in Equation 3. We consider two different cases depending upon whether $\mathbf{n}$ and $\mathbf{n}^{\prime}$ have the same main direction.

1. $\mathbf{n}$ and $\mathbf{n}^{\prime}$ have the same main direction. Let us assume that this direction is $O z$. This condition writes $\mathbf{n}_{z}=\max \left(\left|\mathbf{n}_{x}\right|,\left|\mathbf{n}_{y}\right|,\left|\mathbf{n}_{z}\right|\right)$ and $\mathbf{n}_{z}^{\prime}=\max \left(\left|\mathbf{n}_{x}{ }_{x}\right|,\left|\mathbf{n}_{y}^{\prime}\right|,\left|\mathbf{n}_{z}^{\prime}\right|\right)$. In 


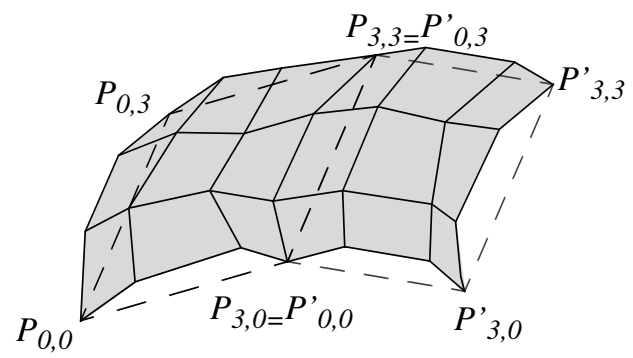

Figure 3: Adjacent bicubic Bézier control nets

that case $Q$ and $Q^{\prime}$ are adjacent and their union is a 26-connected discrete surface iff $Q \cup Q$ ' still has the property of functionality of the individual digital plane patches, which amounts to say that $H \cup H^{\prime}$ has everywhere a thickness of 1 in the direction $\mathrm{Oz}$ and one full side of $H$ and $H^{\prime}$ is contained in their intersection $H \cap H^{\prime}$ (see Figure 4).

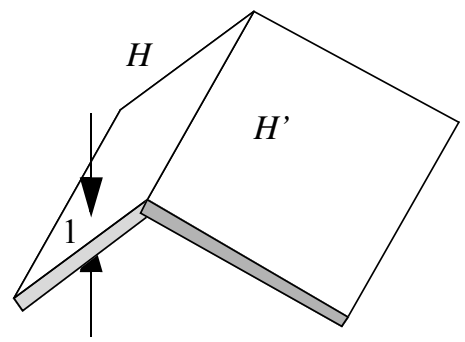

Figure 4: Union of the convex hulls of two discrete plane patches

A problem arises in the case of tensor-product surfaces which may not be planar even though they verify Theorem 3. This results in the choice of inconsistent normal values between adjacent patches that make it difficult to meet the previous connectivity criterion. Such a problem does not occur with triangles, since triangular patches are always planar. Hence, one solution is to divide the tensor-product patches (which are quadrilaterals) into two triangles and work only with discrete triangles.

2. $\mathbf{n}$ and $\mathbf{n}$ ' have different main directions. In the case where $\mathbf{n}$ and $\mathbf{n}$ ' have different main directions the previous condition does not hold anymore and we can only guarantee that $Q \cup Q$ ' is 26-connected if $H \cap H^{\prime}$ ' contains at least one of the sides of $H$ or $H^{\prime}$ and $H \cup H^{\prime}$ has everywhere a thickness of at least 1 with respect to the main directions of $\mathbf{n}$ or $\mathbf{n}$ '.

\section{Conclusion}

In this paper we have presented a method to polygonalize Bézier curves and surfaces into discrete lines and plane patches. Unlike existing rendering algorithms that all require an arbitrary tolerance constant $\varepsilon$, our approach is entirely based on the geometry 
of the manipulated objects and theorems of discrete geometry. Moreover our termination criterion for the subdivision of Bézier curves is optimal. The criterion, though presented here in the case of cubic curves and bicubic surfaces, is general and can be extended to Bézier curves and surfaces of higher degree. Appropriate connectivity of Bézier curves polygonalized by our method is ensured both in 2D and 3D and we also provide a criterion to ensure appropriate connectivity of adjacent discrete surface patches in restricted cases. Further work is needed to determine a more general solution to this connectivity problem of $3 \mathrm{D}$ patches.

\section{References}

1. J.E. Bresenham, Algorithm for Computer Control of a Digital Plotter, IBM Systems Journal, 4(1), 1965, 25-30

2. Sheue-Ling Chang, Michael Schantz and Robert Rochetti, Rendering Cubic Curves and Surfaces with Integer Adaptive Forward Differencing, Computer Graphics, Vol. 23, Nr 3, Jul. 1989, 157-166

3. Gerald Farin, Curves and Surfaces for Computer Aided Geometric Design, A Practical Guide, Academic Press

4. Luiz Henrique de Figueiredo, Adaptive Sampling of Parametric Curves, Graphics Gems V, Academic Press

5. Oscar Figueiredo, Jean-Pierre Reveillès, New Results about 3D Digital Lines, Vision Geometry V, Robert A. Melter, Angela Y. Wu, Longin Latecki, Editors, Proc. SPIE 2826, Aug 96, Denver CO, 98-108

6. James D. Foley, Andries van Dam, Steven K. Feiner and John F. Hughes, Computer Graphics : Principles and Pratice, Addison-Wesley Publishing Company

7. R. Victor Klassen, Intersecting Parametric Cubic Curves by Midpoint Subdivision, Graphics Gems IV, Academic Press, 1994, pp 261-277

8. J.M. Lane and R. F. Riesenfeld, A theoretical development for the computer generation and display of picewise polynomial surfaces, IEEE Trans. Pattern Anal. Machine Intelligence, 2(1), 35-46, 1980

9. Jean-Pierre Reveillès, Géométrie discrète, calcul en nombres entiers et algorithmique, Thèse d'Etat, Université Louis Pasteur, Strasbourg, Dec 1991

10. Bob Wallis, Tutorial on Forward Differencing, Graphics Gems, Andrew S. Glassner Ed., Academic Press

11. Guojin Wang and Wei Xu, The Termination Criterion for Subdivision of the Rational Bézier Curves, CVGIP: Graphical Models and Image Processing, Vol. 53, No. 1, January, pp. 93-96, 1991 